\title{
SÍNDROME DE BURNOUT EN ESTUDIANTES DE MEDICINA DE SANTA ROSA DEL AGUARAY
}

\section{BURNOUT SYNDROME IN MEDICAL STUDENTS OF SANTA ROSA DEL AGUARAY}

\section{José ROJAS-MELGAREJO무, Francisco MERELES-OVIEDO ${ }^{1}$, Iván BARRIOS², Julio TORALES ${ }^{3}$.}

${ }^{1}$ Estudiantes de Medicina, Filial Santa Rosa del Aguaray, Facultad de Ciencias Médicas, Universidad Nacional de Asunción, Santa Rosa del Aguaray - Paraguay.

${ }^{2}$ Estudiante de Matemática Estadística, Facultad de Ciencias Exactas y Naturales, Universidad Nacional de Asunción, San Lorenzo - Paraguay.

33efe de la Cátedra de Socioantropología, Filial Santa Rosa del Aguaray, Facultad de Ciencias Médicas, Universidad Nacional de Asunción, Santa Rosa del Aguaray - Paraguay.

Cómo citar este artículo: Rojas-Melgarejo J, Mereles-Oviedo F, Barrios I, Torales J. Síndrome de Burnout en estudiantes de Medicina de Santa Rosa del Aguaray. Medicina Clínica y Social. 2017;1(1): 26-30.

\section{RESUMEN}

Introducción: El estrés laboral al que están sometidos los profesionales de la salud, junto con el fracaso de los mecanismos de adaptación a situaciones difíciles, lleva a un desgaste emocional y distanciamiento afectivo, componentes del llamado síndrome de Burnout. Objetivo: Determinar la frecuencia de síndrome de Burnout en estudiantes de Medicina de la Filial Santa Rosa del Aguaray, Facultad de Ciencias Médicas, Universidad Nacional de Asunción. Metodología: Estudio descriptivo que incluyó a 61 estudiantes de medicina, se aplicó el Burnout Maslach Inventory para medir síndrome de Burnout. Resultados: Se encontraron altos niveles de despersonalización en el 9,8\%, altos niveles cansancio emocional en el 9,8\% y bajos niveles de realización personal en el 16,4\% de la muestra. La frecuencia encontrada de síndrome de Burnout en los estudiantes de medicina entrevistados fue del 28\%. Discusión: La frecuencia de síndrome de Burnout en nuestra población fue mucho mayor a la esperada según la literatura. Son necesarias más investigaciones longitudinales que busquen identificar factores de riesgo y factores protectores.

Palabras clave: Estrés laboral; Síndrome de Burnout; Estudiantes de Medicina.

\section{ABSTRACT}

Introduction: The work stress to which health professionals are subjected, together with the failure of the mechanisms of adaptation to difficult situations, leads to emotional exhaustion and affective distancing, components of the so-called Burnout syndrome. Objective: to determine the frequency of Burnout syndrome among medical students in the Santa Rosa del Aguaray campus, National University of Asunción School of Medical Sciences. Methodology: Descriptive study that included 61 medical students. The Burnout Maslach Inventory was applied in order to measure Burnout syndrome. Results: High levels of depersonalization were found in 9.8\%, high levels of emotional exhaustion in $9.8 \%$ and low levels of personal performance in $16.4 \%$ of the sample. The frequency of Burnout syndrome in the interviewed Medical students was $28 \%$. Discussion: The frequency of Burnout syndrome in our population was much higher than expected according to the literature. More longitudinal research is needed to identify risk and protective factors.

Keywords: Work stress; Burnout syndrome; Medical students. 


\section{INTRODUCCIÓN}

El estrés laboral al que están sometidos los profesionales de la salud, junto con el fracaso de los mecanismos de adaptación a situaciones difíciles, lleva a un desgaste emocional y distanciamiento afectivo, componentes del llamado síndrome de Burnout (1). La ansiedad y la depresión son factores presentes en un alto porcentaje de estudiantes universitarios, sobre todo de Medicina, y han sido objeto de investigaciones previas que han demostrado su mayor presencia que en la población general (2-4). Lo anterior puede ser un detonante para el desgaste emocional en la vida laboral. Asimismo, se describen otros factores que pueden llegar a influir en el estado mental de los estudiantes, tales como la autoestima y la inteligencia emocional $(5,6)$, entre otros.

El estudio del estrés laboral es de suma importancia, pues influye no solo en el desempeño de las personas sino también en su salud mental. Aquí radica la importancia de estudiar este fenómeno ya en los primeros años de la carrera de Medicina, con el fin de realizar intervenciones para disminuir los factores estresores o aprender a enfrentarlos. El síndrome de Burnout se presenta en los estudiantes de Medicina con una frecuencia entre 1-22\% y uno de los principales desafíos en la investigación de este síndrome es poder reconocer los factores de riesgo y aquellos que son protectores (7).

Con base en lo anterior, se ha constituido en el objetivo principal de esta investigación determinar la frecuencia de síndrome de Burnout en la población constituida por los estudiantes de Medicina de la Filial de Santa Rosa del Aguaray de la Facultad de Ciencias Médicas, de la Universidad Nacional de Asunción, que se encuentra en el Departamento de San Pedro, norte de Paraguay.

\section{METODOLOGÍA}

Fue un estudio descriptivo de corte transversal, no probabilístico de casos consecutivos, llevado a cabo en la ciudad de Santa Rosal del Aguaray, Departamento de San Pedro, durante junio de 2016. La población enfocada estuvo constituida por todos los estudiantes de medicina de la Facultad de Ciencias Médicas, Filial Santa Rosa del Aguaray, Universidad Nacional de Asunción; mientras que la población accesible la constituyeron los estudiantes que aceptaron participar de forma voluntaria de la investigación. Se excluyó a aquellos estudiantes que no completaron en forma correcta el cuestionario o que dejaron campos sin responder.

Con base en una frecuencia de $22 \%$ (7), una precisión del $11 \%$ y un intervalo de confianza del $95 \%$, se obtuvo un tamaño muestral mínimo de 54 sujetos; finalmente pudo obtenerse la respuesta de 61 estudiantes que cumplieron con los criterios de inclusión.

Se aplicó el Burnout Maslach Inventory, para medir síndrome de Burnout, elaborado con base en una escala ordinal del tipo Likert ("nunca", "algunas veces al año", "algunas veces al mes", "algunas veces a la semana" y "diariamente"; con valores ascendentes del 1 al 5, respectivamente). El Burnout Maslach Inventory está conformado por 22 preguntas distribuidas en tres subescalas: despersonalización (D), agotamiento emocional ( $A E$ ) y realización personal (RP). La subescala de despersonalización está formada por cinco preguntas relacionadas con una respuesta impersonal y falta de sentimientos hacia los sujetos que son objeto de atención (por ejemplo, los pacientes para médicos y estudiantes de Medicina); la subescala de agotamiento emocional está formada por nueve preguntas relacionadas con agotamiento laboral; mientras 
que la subescala de realización personal está formada por ocho preguntas que valoran sentimientos de competencia y realización exitosa en el trabajo hacia los demás (8).

Se consideran 3 categorías: nivel bajo para los individuos encontrados por debajo del percentil 33 , nivel medio para los encontrados entre los percentiles 33 y 66 , y nivel alto para aquellos encontrados por encima del percentil 66. El criterio para el diagnóstico del síndrome de Burnout es la obtención de un nivel alto en las escalas de despersonalización y agotamiento emocional, y un nivel bajo en la escala de realización personal (8).

Los datos obtenidos fueron archivados utilizando tablas de Microsoft Excel ${ }^{\circledR}$ y posteriormente analizados con el software estadístico Epilnfo versión 7.2 (CDC, Atlanta). Se garantizó el anonimato de los estudiantes de Medicina al momento de la toma de muestras, así como durante el análisis y la presentación de los resultados. Solo participaron estudiantes que aceptaron hacerlo en forma voluntaria, luego de haber conocido el propósito de la investigación.

\section{RESULTADOS}

La muestra estuvo conformada por 61 universitarios, de los cuales $64 \%$ fueron del sexo femenino, con edades comprendidas entre los 17 y 33 años (media de $22 \pm 4$ años). Los aspectos estudiados con la escala fueron: despersonalización, realización personal y agotamiento emocional; hablamos de presencia de síndrome de Burnout cuando nos enfrentamos a altos niveles de despersonalización y agotamiento emocional, y bajos niveles en la subescala de realización personal.

Los datos referentes a estos tres aspectos pueden encontrarse en la Tabla 1, donde resaltan altos niveles de despersonalización en el $9,8 \%$, altos niveles de agostamiento emocional en el $9,8 \%$ y bajos niveles de realización personal en el $16,4 \%$ de la muestra. Con estos datos podemos expresar que la frecuencia encontrada de síndrome de Burnout en los estudiantes de Medicina entrevistados fue del $28 \%$ (17 estudiantes).

TABLA 1. FRECUENCIAS ENCONTRADAS EN LAS SUBESCALAS DEL BURNOUT MASLACH INVENTORY

\begin{tabular}{|c|c|c|c|}
\hline Niveles & Despersonalización & Agotamiento emocional & Realización personal \\
\hline Bajo & $25(41 \%)$ & $22(36,1 \%)$ & $10(16,4 \%)$ \\
Medio & $30(49,2)$ & $33(54,1 \%)$ & $28(45,9 \%)$ \\
Alto & $6(9,8)$ & $6(9,8 \%)$ & $23(37,7 \%)$ \\
& & & \\
\hline
\end{tabular}

\section{DISCUSIÓN}

El síndrome de Burnout va en aumento en los profesionales de la salud, así como en los estudiantes universitarios del área; este fenómeno puede repercutir en el desempeño, calidad y seguridad de la atención a los pacientes, allí la importancia de su detección precoz ya desde el grado (9). 
Entre todos los profesionales de la salud, la frecuencia de síndrome de Burnout encontrada en médicos es del 3,7\%, mientras que en los enfermeros es de $2,1 \%$ (10). La amplia diferencia encontrada entre estas cifras y las emanadas de la presente investigación nos obliga a pensar en posibles sesgos que pudieron haber afectado la toma de muestra, como por ejemplo, una de las limitaciones fue no contar con un muestreo probabilístico que nos permita generalizar nuestros hallazgos. También debemos tener en cuenta en qué momento se tomó la muestra, ya que, si los estudiantes se encontraban cercanos a exámenes, esta situación pudo haber influido en las respuestas (11).

Debería investigarse cuáles son los factores que influyen o están relacionados con este síndrome, ya que son varias las publicaciones que informan la frecuencia encontrada en diversas poblaciones; asimismo, es fundamental tener en cuenta que en la población universitaria puede necesitarse de escalas diferentes a las aplicadas en la población general, teniendo en cuenta que estas escalas fueron diseñadas para medir el estrés laboral y no necesariamente el académico.

Además de identificar este síndrome, hay que enfocarse en verificar de qué subtipo específico se trata y qué patologías acompañan a estos signos y síntomas; esto principalmente para abordar el tratamiento, ya que las nuevas intervenciones se centran en enfrentar los sentimientos de desesperanza y falta de eficacia, pudiendo así recuperar la confianza (12).

Podemos concluir que el síndrome de Burnout es una entidad frecuente en los estudiantes de Medicina de la Filial Santa Rosa del Aguaray de la Facultad de Ciencias Médicas de la Universidad Nacional de Asunción, y que son necesarias más investigaciones longitudinales que busquen establecer relaciones con variables, identificando factores de riesgo y factores protectores. Lo anterior ayudará a realizar intervenciones tempranas tendientes a prevenir esta entidad y favorecer el pasaje de los estudiantes a través del grado de la, preparándose de esa manera para enfrentar los estresores que se presentarán en la vida laboral.

\section{CONFLICTOS DE INTERÉS Y FUENTE DE FINANCIACIÓN}

Los autores declaran no poseer conflictos de interés. Fuente de financiación: ninguna.

\section{REFERENCIAS BIBLIOGRÁFICAS}

1. Mariños A, Otero M, Tomateo J, Málaga G. Coexistencia de síndrome de Burnout y síntomas depresivos en médicos residentes. Estudio descriptivo transversal en un hospital nacional de Lima. Rev Medica Hered. 2011;22(4):162-168. URL.

2. Barrios I, Miltos V, Piris A, Piris G, Ramírez C, Rodríguez J, et al. Tamizaje de salud mental mediante el test M.I.N.I. en estudiantes del ciclo básico de medicina de la Universidad Nacional de Asunción. An Fac Cienc Méd. 2015;48(1):59-68. DOI: 10.18004/anales/2015.048(01)59-068

3. Torales Benítez J, Girala N, Moreno Giménez M, Arce A, Trinidad S, Estigarribia E, et al. Depresión y ansiedad en estudiantes de medicina de la Universidad Nacional de Asunción. Rev Par Psiquiatr. 2013;1(1):12-28. URL.

4. Barrios I, Piris A, Ruiz-Díaz C, Rodríguez Andersen G, Torales J. Niveles de depresión y ansiedad y su relación con la presencia de alexitimia en estudiantes universitarios de 
la Facultad Politécnica de la Universidad Nacional de Asunción. Rev Latinoam Psiquiatr. 2015;16(2):7-14. URL.

5. Barrios I, Piris A, Torales J, Viola L. Autoestima en estudiantes de medicina de la Universidad Nacional de Asunción, Paraguay. An Fac Cienc Méd. 2016;49(2):27-31. DOI: 10.18004/anales/2016.049(02)27-032

6. Ayala Servín N, Bogarín Torres C, Bottrell A, Duarte Fariña C, Torales J, Samudio M, et al. Inteligencia emocional y coeficiente intelectual como predictor de rendimiento académico en estudiantes de medicina paraguayos: Año 2015. Mem Inst Investig Cienc Salud. 2016;14(2):84-91. DOI: 10.18004/Mem.iics/1812-9528/2016.014(02)92-097

7. Mejia CR, Valladares-Garrido MJ, Talledo-Ulfe L, Sánchez-Arteaga K, Rojas C, Arimuya $\mathrm{JJ}$, et al. Síndrome de Burnout y factores asociados en estudiantes de medicina. Estudio multicéntrico en siete facultades de medicina peruanas. Rev Chil Neuro-Psiquiatr. 2016;54(3):207-214. DOI: 10.4067/S0717-92272016000300005

8. Gil-Monte PR. Validez factorial de la adaptación al español del Maslach Burnout Inventory-General Survey. Salud Pública Méx. 2002;44(1):33-40. URL.

9. Terrones-Rodríguez JF, Cisneros-Pérez V, Arreola-Rocha JJ. Síndrome de burnout en médicos residentes del Hospital General de Durango, México. Rev Med Inst Mex Seguro Soc. 2016;54(2):242-248. URL.

10. Maticorena-Quevedo J, Beas R, Anduaga-Beramendi A, Mayta-Tristán P. Prevalencia del síndrome de Burnout en médicos y enfermeras del Perú, Ensusalud 2014. Rev Peru Med Exp Salud Publica. 2016;33(2):241-247. DOI: 10.17843/rpmesp.2016.332.2170

11. Torales J, Troche Z, Ibarrola E, Micó G, Barrios I, Bolla L, et al. Subjective psychological wellbeing in medical students: a pilot study from Paraguay. Int J Cult Ment Health. 2016;9(4):373-380. DOI: 10.1080/17542863.2016.1218522

12. Montero-Marín J. El síndrome de Burnout y sus diferentes manifiestaciones clínicas: una propuesta para la intervención. Anest Analg Reanim. 2016;29(1). URL. 